\title{
Future perspectives for breast conservation treatment: science \& art
}

\section{Introduction}

There are two components to breast conservation treatment (BCT): science and art. Science involves statistical interrogation of outcomes between a limited number of treatment strategies. For the surgeon, 'art' comprises integration of available clinical information that leads to decision making with respect to operative techniques applied to complete the surgical phase of treatment. Both fields are interdependent and overlap to a certain degree but it is also possible to separate them into two different realms. In each sphere, one aspect dominates over the other. Surgical oncology presents a unique circumstance, where the art of the treatment form with the same term lies in the hands of an individual, the surgeon. In other words, the same term BCT can take different forms when two different surgeons operate. The same is not the case with medical oncology, where the drug of the same name prescribed by two different clinicians has the same constituents.

The 'science' of BCT tends to exclude how surgery is performed. This involves comparing the outcomes of two different types of treatment in terms of statistics. The data acquired informs optimum therapy. The 'art' of BCT encompasses an entire spectrum, and ranges from standard breast conserving surgery (sBCS) with or without parenchymal closure to therapeutic mammoplasty and volume replacement procedures. It also involves the patient's perspective on that final result and her quality of life. How each interacts and influences the other will determine what form BCT takes in the future.

\section{The science of BCT}

In the era of the Halsted radical mastectomy, the 'how' (art) of surgery dominated the science of surgery. The operation he described in 1894 involved resection of the breast, axillary lymph nodes and pectoralis muscles. He recorded a 5-year survival of $40 \%$ for treated women, which was twice that for untreated women (1). However, survivors had significant ipsilateral arm morbidity, lymphoedema and a grotesque deformity. Subsequent to that, Patey et al. introduced a less mutilating operation sparing the pectoralis muscles, and compared results with the radical mastectomy (2). There was no difference in survival or local recurrence between the two groups. Although there was a move towards decreasing surgical morbidity, mastectomy continued to be the primary mode of treatment for breast cancer until the last quarter of the twentieth century when Veronesi and Fisher led separate teams comparing it with BCT $(3,4)$. The science of these seminal randomised controlled studies ushered in the epoch of BCT.

It would be simplistic to state that this archetypal form of surgical de-escalation was the panacea for the morbidity and body-image difficulties that resulted from mastectomy. It raised a host of issues previously unencountered. One of them was the deformity occurring in $30 \%$ of women who completed BCS with radiotherapy (5). This was the consequence of resecting a significant portion of breast tissue without manoeuvres to correct the resulting parenchymal defect. Concurrently, surgical margins became a cause for concern as positive margin status was found to be associated with poorer local control, which potentially had implications for survival (6). These competing demands served as the impetus for research firstly, to define the optimum margin width, and secondly, to develop techniques which would prevent parenchymal distortion. The former culminated in a consensus guideline recommending 'no ink on tumour' as the standard requirement for invasive carcinoma (7), with $2 \mathrm{~mm}$ margins recommended for ductal carcinoma in situ (DCIS) (8). Poor aesthetic results became the driver for the establishment of a myriad of surgical approaches collectively termed as 'oncoplastic breast surgery' (OBS), which forms the 'art' of BCT and will be discussed in the following section.

Achieving the requisite margin status while minimising re-excision is another area for which ongoing research is undertaken. Many techniques are used for margin assessment. Conventional means like frozen section analysis, imprint cytology, intraoperative ultrasonography are compared against more innovative methods like radiofrequency spectrometry, optical coherence tomography and radioactive seed localisation $(9,10)$. However, there is no clearly superior approach at present and this area needs to be further explored (9).

Several authors in this issue have offered insights into the science of BCT, its historical development and how it is applied in treatment today (11-13). However, the science continues to evolve as evidence is presented to allow best practice according 
to the contemporary 'state of the art'. For example, recent step-wise advances in knowledge have diminished the influence of conditions like inflammatory breast cancer and multifocal, multicentric tumour as contraindications to BCT. With appropriate selection, patients with these disease types may now undergo BCS provided certain conditions are fulfilled $(14,15)$. Aside from the local treatment of the breast, axillary dissection is no longer considered to be standard of care for early breast cancers with clinically negative nodes. Sentinel node biopsy alone may also suffice in selected cases of patients with minimal disease burden in the axilla $(16,17)$. It is not inconceivable that other 'stalwart' treatment approaches for breast cancer will undergo a paradigm shift in the future with the increasing accumulation of data to inform surgical practice.

Breast cancer treatment has entered the genomic era, and this is likely to take a central role in research moving forward. Molecular signatures are already routinely used to inform medical therapy (18). Through a better understanding of carcinogenesis based on the sick lobe hypothesis and the genetic changes identified on a cellular level, it may be possible in future to use molecular characteristics to determine optimal resection volumes (19-21). There is a high possibility that in the near future, genomics will be used as part of the selection criteria for BCT-eligibility. This is an exciting area for further research, for it will allow appropriate de-escalation of surgical therapy while selecting patients who will need treatment intensification. Work in this area will enable breast cancer treatment to move into the age of personalised or precision surgery.

\section{The art of BCT}

As alluded to in the foregoing section, the science for breast cancer treatment has brought about the art associated with it. BCT, while allowing a de-escalation of treatment from a previous standard of mastectomy, resulted in approximately onethird of patients having a significant deformity (22). This is a unique feature of breast cancer treatment where no obvious functional deficit ensues but there is a quality of life issue which requires resolution. It was argued more than a decade ago that optimal surgical treatment should anticipate the potential for deformity and preventive action should be taken during the primary surgery (22). This call for pre-emptive measures led to the introduction and integration of plastic and reconstructive operative techniques into tumour resection plans, known now as OBS. There is a wide array of these techniques which have been described. Different institutions and surgeons have developed their own methods of achieving good cosmetic outcomes, with some proposing 'extreme oncoplasty' (23-25). However, there is contradictory evidence with respect to patient reported outcomes, local control and surgical complications and available data does not offer convincing evidence that OBS is superior to sBCS (26-29). Hence the call for aesthetic outcomes to take precedence over adequate and appropriate treatment for breast cancer (30) cannot be of primary importance (31). Care should be taken to guard against overtreatment. The trend towards more extensive surgery with OBS may in fact be supererogatory for oncologic control. The objective of surgical treatment should still be to achieve the highest survival and best local control with the lowest morbidity. Hence, a reductionist, or minimalist approach to fulfil oncologic requirements may provide optimal results $(32,33)$. Such an approach would require an integration of appropriate preoperative imaging, careful surgical planning and detailed pathologic examination $(34,35)$. This is consistent with the argument by Marescaux et al. that iatrogenic impact should be reduced in surgical innovation for patient safety and quality of life (36).

Unfortunately, it is extremely difficult, if not impossible, to satisfactorily derive a method to determine the optimal surgical approach, whether OBS or sBCS. For unlike physicians, surgeons cannot be 'blinded' when conducting studies for surgical techniques. Such analyses will always be confounded by individual procedural and technical biases which cannot be completely eradicated or accounted for. There is no 'one size fits all' surgical approach for BCT, and each surgeon may have particular preferences based on his or her training and treatment philosophy. There is also a need to explore communication strategies in the light of the multitude of surgical techniques for BCT for patient decision making. The challenges presented herein may be an area for future research.

\section{Discussion}

It is evident that in BCT, the science blends with the art. While there is a distinction between the two, each entity drives the other, and both meld into a complex whole which needs to be grasped and interpreted in the context of the individual patient for clinical decision making with respect to optimal therapy. With the mounting volume of data now available, this 
task becomes increasingly daunting for the physician. It is conceivable that artificial intelligence may be called upon to assist in clinical decision making and possibly take over in part the role of the surgeon, as has been suggested in the realm of multidisciplinary approaches for drug therapy (37). This scenario may create a greater need for the clinician to adapt to advances in technology, and perhaps create a new niche in the healthcare sphere. The breast surgeon of the future will have many more considerations to undertake than his predecessor practising two decades in the past, but for the present, it is still within his power to lead research and direct surgical therapy in the path that would result in the most favourable outcomes for the patient with breast cancer.

\section{Acknowledgements}

None.

\section{References}

1. Halsted WS. The results of operations for the cure of cancer of the breast performed at the Johns Hopkins Hospital from June 1889 to January 1894. Ann Surg 1894;20:497-555.

2. Patey DH, Dyson WH. The prognosis of carcinoma of the breast in relation to the type of the mastectomy performed. Br J Cancer 1948;2:7-13.

3. Veronesi U, Cascinelli N, Nariani L, et al. Twenty-year follow-up of a randomized study comparing breast-conserving surgery with radical mastectomy for early breast cancer. N Engl J Med 2002;347:1227-32.

4. Fisher B, Anderson S, Bryant J, et al. Twenty-year follow-up of a randomised trial comparing mastectomy, lumpectomy, and lumpectomy plus irradiation for the treatment of invasive breast cancer. N Engl J Med 2002;347:1233-41.

5. Clough KB, Cuminet J, Fitoussi A, et al. Cosmetic sequelae after conservation treatment for breast cancer: classification and results of surgical correction. Ann Plast Surg 1998;41:471-81.

6. Peterson ME, Schultz DJ, Reynolds C, et al. Outcomes in breast cancer patients relative to margin status after treatment with breast-conserving surgery and radiation therapy: the University of Pennsylvania experience. Int J Radiat Oncol Biol Phys 1999;43:1029-35.

7. Moran MS, Schnitt SJ, Giuliano AE, et al. Society of Surgical Oncology-American Society for Radiation Oncology consensus guideline on margins for breast-conserving surgery with whole-breast irradiation in stages I and II invasive breast cancer. Ann Surg Oncol 2014;21:704-16.

8. Morrow M, Van Zee KJ, Solin LJ, et al. Society of Surgical Oncology-American Society for Radiation Oncology-American Society of Clinical Oncology consensus guideline on margins for breast-conserving surgery with whole-breast irradiation in ductal carcinoma in situ. Ann Surg Oncol 2016;23:3801-10.

9. Butler-Henderson K, Lee AH, Price RI, et al. Intraoperative assessment of margins in breast conserving therapy: a systematic review. Breast 2014;23:112-9.

10. Ahmed M, Douek M. Radioactive seed localisation (RSL) in the treatment of non-palpable breast cancers: a systematic review and meta-analysis. Breast 2013;22:383-8.

11. McEvoy MP, Landercasper J, Naik HR, et al. Update of the American Society of Breast Surgeons Toolbox to address the lumpectomy reoperation epidemic. Gland Surg 2018;7:536-53.

12. Murugappan K, Saboo A, Kuo L, et al. Paradigm shift in the local treatment of breast cancer: mastectomy to breast conservation surgery. Gland Surg 2018;7:506-19.

13. Boyages J, Baker L. Evolution of radiotherapy techniques in breast conservation treatment. Gland Surg 2018;7:576-95.

14. Brzezinska M, Dixon JM. Inflammatory breast cancer: no longer an absolute contraindication for breast conservation surgery following good response to neoadjuvant therapy. Gland Surg 2018;7:520-24.

15. Winters ZE, Bernaudo L. Evaluating the current evidence to support therapeutic mammoplasty or breast-conserving surgery as an alternative to mastectomy in the treatment of multifocal and multicentric breast cancers. Gland Surg 2018;7:525-35.

16. Krag DN, Anderson SJ, Julian TB, et al. Sentinel ymph node resection compared with conventional axillary lymph node 
dissection in clinically node-negative patients with breast cancer: overall survival findings from the NSABP B-32 randomised phase 3 trial. Lancet Oncol 2010;11:927-33.

17. Giuliano AE, Ballman K, McCall L, et al. Locoregional Recurrence After Sentinel Lymph Node Dissection With or Without AxillaryDissection in Patients With Sentinel Lymph Node Metastases: Long-term Follow-up From the American College of Surgeons Oncology Group (Alliance) ACOSOG Z0011 Randomized Trial. Ann Surg 2016;264:413-20.

18. Wong NS. Primary medical therapy and breast conservation treatment: the medical oncology perspective. Gland Surg 2018;7:560-75.

19. Tot T. DCIS, cytokeratins, and the theory of the sick lobe. Virchows Arch 2005;447:1-8

20. Lebya K, Garcia-Smith R, Swaminathan R, et al. Towards a personalised surgical margin for breast conserving surgery implications of field cancerisation in local recurrence. J Surg Oncol 2017;115:109-15

21. Desmedt C, Fumagalli D, Pietri E, et al. Uncovering the genomic heterogeneity of multifocal breast cancer. J Pathol 2015;236:457-66.

22. Baildam AD. Oncoplastic surgery of the breast. Br J Surg 2002;89:532-33.

23. Clough KB, Kaufman GJ, Nos C, et al. Improving breast cancer surgery: a classification and quadrant per quadrant atlas for oncoplastic surgery. Ann Surg Oncol 2010;17:1375-91.

24. Kronowitz SJ, Kuerer HM, Buchholz TA, et al. A management algorithm and practical oncoplastic surgical techniques for repairing partial mastectomy defects. Plast Reconstr Surg 2008;122:1631-47.

25. Silverstein MJ. Radical mastectomy to radical conservation (extreme oncoplasty): a revolutionary change. J Am Coll Surg 2016;222:1-9.

26. Carter SA, Lyons GR, Kuerer HM, et al. Operative and oncologis outcomes in 9861 patients with operable breast cancer: single-institution analysis of breast conservation with oncoplastic reconstruction. Ann Surg Oncol 2016;23:3190-8.

27. De Lorenzi F, Hubner G, Rotmensz N, et al. Oncological results of oncoplastic breast-conserving surgery: long term follow-up of a large series at a single institution: a matched-cohort analysis. Eur J Surg Oncol 2016;42:71-7.

28. Acea Nebril B, Garea CC, Novoa AG. Cosmetic sequelae after oncoplastic surgery of the breast. Classification and factors for prevention. Cir Esp 2015;93:75-83

29. Mattingly AE, Ma Z, Smith PD, et al. Early postoperative complications after oncoplastic reduction. South Med J 2017;110:660-6.

30. Carmichael AR, Mokbel K. Evolving trends in breast surgery: Oncoplastic to Onco-Aesthetic Surgery. Arch Plast Surg 2016;43:222-3.

31. Catanuto G, Rocco N, Nava MB. De-escalating oncoplastic breast surgery. Minerva Chir 2018;73:300-2

32. Tan MP. Toward a Reductionist Approach to the Surgical Treatment of Breast Cancer. J Am Coll Surg 2016;222:967.

33. Tan MP. Minimalist breast conserving surgical approaches for inferiorly sited cancers. Gland Surg 2017;6:399-409.

34. Ong E. Preoperative imaging for breast conservation surgery—do we need more than conventional imaging for local disease assessment? Gland Surg 2018;7:554-59.

35. Tan MP, Tot T. The sick lobe hypothesis, field cancerisation and the new era of precision breast surgery. Gland Surg 2018;7:611-8.

36. Marescaux J, Diana M. Inventing the future of surgery. World J Surg 2015;39:615-22.

37. Lin FP, Pokorny A, Teng C, et al. Computational prediction of multidisciplinary team decision-making for adjuvant breast cancer drug therapies: a machine learning approach. BMC Cancer 2016;16:929. 


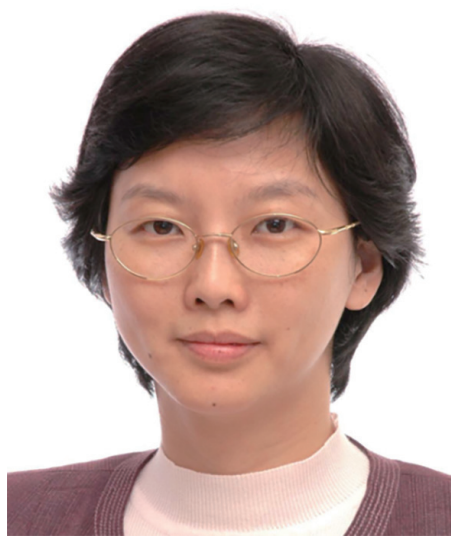

Mona P. Tan

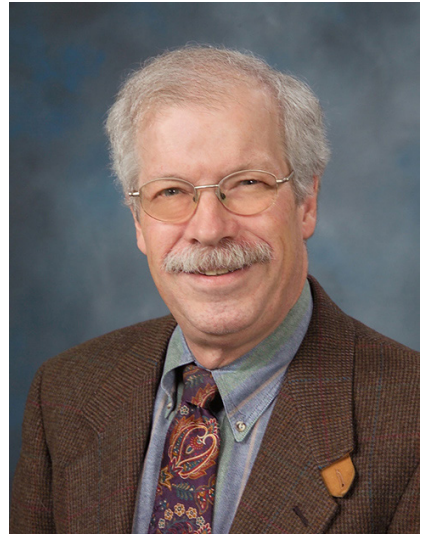

Edibaldo Silva

Mona P. Tan

Breast Surgeon, MammoCare, Singapore, Singapore. (Email: jabezhopems@gmail.com)

Edibaldo Silva

Department of Surgery, University of Nebraska Medical Centre, Nebraska Medical Centre, Omaha, NE, USA.

(Email: esilva@unmc.edu) doi: 10.21037 /gs.2018.12.03

Conflicts of Interest: The authors have no conflicts of interest to declare.

View this article at: http://dx.doi.org/10.21037/gs.2018.12.03

Cite this article as: Tan MP, Silva E. Future perspectives for breast conservation treatment: science \& art. Gland Surg 2018;7(6):501-505. doi: 10.21037/gs.2018.12.03 